\title{
Three-Dimension Random Aggregate Generation of Numerical Concrete Model
}

\author{
Jianxin Ding ${ }^{1}$ and Qingzhou Yang ${ }^{2}$ \\ ${ }^{1}$ Changjiang Institute of Survey, Planning, Design and Research, Wuhan, 430010, China \\ ${ }^{2}$ PowerChina Hubei Electric Engineering Corporation Limited, Wuhan, 430040, China
}

\begin{abstract}
The aggregate generation of concrete is one of the important problems in concrete mesoscopic mechanics. Firstly, the mesoscopic numerical model with spherical aggregates is obtained by the method of excluding the occupied space, and fully-graded concrete model of high aggregate content can be quickly generated. Then, based on the spherical aggregate model, the generation method of random convex polyhedral aggregates is proposed. Finally, a full-graded concrete model with spherical aggregates is shown in Case 1 and a cylindrical concrete model with random convex polyhedral aggregates is shown in Case 2 . The result shows that the aggregates are equally distributed in the concrete models which can be used in the study of mesoscopic numerical calculation.
\end{abstract}

\section{Introduction}

Concrete is a kind of typical heterogeneous composite material. Mesoscopic mechanics is one of the important methods of property research of concrete material, which has distinct advantages. The concrete mesoscopic mechanics method mainly includes: the generation and packing of aggregates, mesh discretization, and the numerical analysis of the mesoscopic mechanics model. These three aspects are the main contents of the mesoscopic mechanics, and also the major difficulties. Among them, the former two aspects are the basis of mesoscopic mechanics analysis, the generation and packing of aggregates with high content is difficult, while the aggregate content is very high in the actual hydraulic structures of mass concrete. At present the studies mainly focus on two-dimensional plane problem with circular or polygonal aggregates, while little three-dimensional research with spherical or polyhedral aggregates.

Scholars proposed various methods to generate and pack concrete aggregates, and achieved a series of research results. Wittmann earlier proposed a generation method based on natural aggregate shape, which promotes the development of the concrete mesoscopic modeling [1]. Yue generated two-dimensional asphalt concrete mesoscopic structure model using digital image processing technology [2]. Ma proposed the method of excluding the occupied space to generate threedimensional sphere and polyhedron aggregate model [3]. Cusatis developed the three-dimensional lattice discrete particle mesoscopic model, the spherical aggregate generated according to the aggregate volume fraction, and the geometrical topology algorithm is adopted to pack the aggregates [4]. Wang put forward one two- dimensional modeling approach of concrete aggregates with arbitrary shapes based on a pre-fabricated mesh [5].

In this paper, the generation method of the random aggregates of concrete is studied. Firstly, the method of excluding the occupied space is used to generate and pack spherical aggregates, and the concrete mesoscopic mechanics model with high content of aggregates can be quickly obtained. Then, a novel method is proposed for the generation of the random convex polyhedral aggregates. The generation program is compiled. Finally, A $450 \mathrm{~mm} \times 450 \mathrm{~mm} \times 450 \mathrm{~mm}$ full-graded concrete cube model with random spherical aggregates is shown in Case 1 and a cylindrical concrete model with random convex polyhedral aggregates is shown in Case 2, The results show that the aggregates are equally distributed in the concrete models which can be used in the study of mesoscopic numerical calculation.

\section{The generation of random spherical aggregates}

In concrete mesoscopic mechanics research, the aggregate is mostly assumed as spherical or polyhedral aggregate (circular or polygonal aggregate in twodimensional model). Due to the intrusive contact judgment between aggregates is simple, the achievements mainly focus on the circular and spherical shape for aggregates. The generation and packing of threedimensional spherical aggregates is relatively simple, but the actual aggregate content is low in a lot of papers, which did not consider the actual aggregate gradation, and most of the actual concrete aggregate content is about $40 \%$ 60\%, while in mass concrete of hydraulic 
structures, the content of aggregates is even higher, the generation and packing of concrete aggregates with fullgraded and high content of the concrete mesoscopic mechanics is one of the problems to be solved. Ma proposed "the method of excluding the occupied space", which can more effectively finish high content aggregate generation and packing in certain space [3]. Qing studied the three-dimensional mesoscopic mechanics model of concrete based on CT slice [6]. This paper adopts the method of excluding the occupied space to generate concrete aggregates, and all aggregates are regarded as coarse aggregates, while the fine aggregates are supposed to be mortar.
As the method of excluding the occupied space, the specimen area is divided into cube cell with a step length less than the minimum aggregate radius, when packing aggregates, the element whose maximum distance from nodes to the aggregate center is less than the radius plus the minimum aggregate radius, the element will be excluded. When determining the position of the rest of the aggregates, the element excluded will not be considered. The method can improve the efficiency of the three-dimensional random spherical aggregate generation [3]. The algorithm is shown in Figure 1.

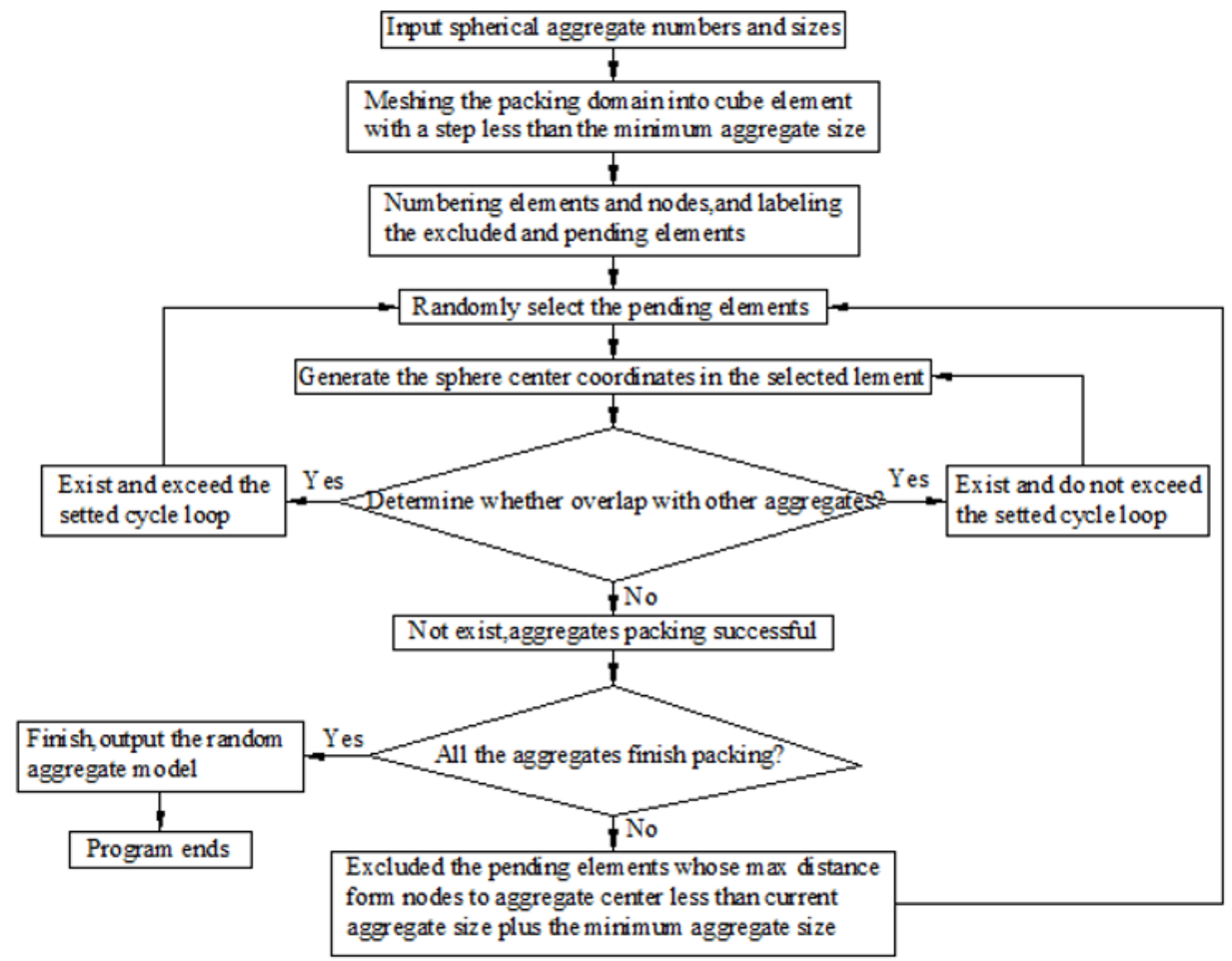

Figure 1. The program flow chart of $3 \mathrm{D}$ random spherical aggregate generation and packing algorithm.

\section{The generation of random convex polyhedral aggregates}

The shape of the artificial crushed aggregates is mainly convex polyhedron. In order to reflect the irregular shape of the aggregates, the random convex polyhedral particle is generated in the ellipsoid (Figure 2). Reference to the stone particles of rockfill dam generation method proposed by Ma [7], the random vertices are generated on the ellipsoid surfaces, the number of vertices is stochastic, and then the convex polyhedron is generated by the vertices according to the certain algorithm, the specific method is as follows:

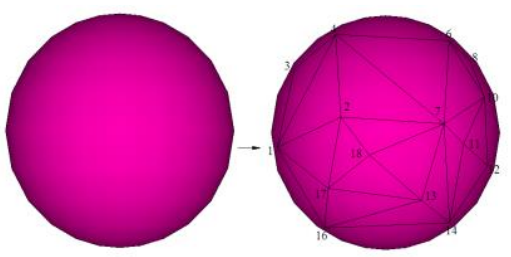

Figure 2. The ellipsoidal aggregate model.
Firstly, the radius of the ellipsoid is obtained on the basis of the generated spherical aggregate:

$$
\left\{\begin{array}{l}
a=r_{1}+\left(r_{2}-r_{1}\right) * R A N D(1) \\
b=r_{1}+\left(r_{2}-r_{1}\right) * R A N D(2) \\
c=r_{1}+\left(r_{2}-r_{1}\right) * R A N D(3)
\end{array}\right.
$$

where: $a 、 b$ and $c$ are the length of the three semi-axis of the ellipsoid; $r_{1}$ is the lower limit of a certain size group; $r_{2}$ is the upper limit of the size group, $R A N D$ (1)

$R A N D$ (2) and $R A N D(3)$ are independent random numbers distributed equally in the interval $[0,1]$.

In order to ensure that the shape of the aggregates is fully random, the number of vertices is equally distributed:

$$
n=n_{\text {min }}+\left(n_{\text {max }}-n_{\text {min }}\right) * R A N D
$$


where: $R A N D$ is the random number which is equally distributed in the interval $[0,1]$. In this paper, the $n_{\min }$ is set to 10 and $n_{\max }$ is 18 .

The vertex coordinates of the convex polyhedron is calculated by spherical coordinates:

$$
\left\{\begin{array}{l}
x_{i}=x_{0}+a \sin \theta_{i} \cos \varphi_{i} \\
y_{i}=y_{0}+b \sin \theta_{i} \sin \varphi_{i} \\
z_{i}=z_{0}+c \cos \theta_{i}
\end{array}\right.
$$

where: $\left(x_{i}, y_{i}, z_{i}\right)$ is the coordinate of the vertice $i$ in the ellipsoid surface; $\left(x_{0}, y_{0}, z_{0}\right)$ is the center coordinate of the ellipsoid; $\theta_{i}$ is the dip angle in the Spherical Coordinate System, which is of the uniform distribution in the interval $[0, \pi] . \varphi_{i}$ is the direction angle in the Spherical Coordinate System and equally distributed in the interval $[0,2 \pi]$.

In this paper, a novel method is proposed for the generation of the convex polyhedral aggregates with randomly selected vertices. After the number of the vertices and the coordinates of the vertices are determined, three vertices in the vertices set are randomly selected, then a plane is determined by the three vertices, and the equation of the plane is calculated. If the other vertices in addition to the three vertices are on the same side of the plane, the plane is an effective surface for the convex polyhedron, a convex polyhedron aggregate is generated until all the effective surfaces are find out.
There are sharp angles and thin deformed aggregates when using the above algorithm to generate random convex polyhedrons. It needs to improve the generation of convex polyhedral vertices. Specifically, the main control is the generation intervals for $\theta_{i}$ and $\varphi_{i}$. When the number of vertices of the convex polyhedron aggregate is determined, the generation of the interval $[0$, $\pi],[0,2 \pi]$ are divided into $n$ sub-intervals, the $\theta_{i}$ and $\varphi_{i}$ are equally distributed in the interval $\left[\frac{\pi}{n}(i-1), \frac{\pi}{n} i\right]$ and $\left[\frac{2 \pi}{n}(i-1), \frac{2 \pi}{n} i\right]$, the shape of the generated convex polyhedral aggregate is more regular.

\section{Examples}

Case 1: The $450 \mathrm{~mm} \times 450 \mathrm{~mm} \times 450 \mathrm{~mm}$ full-graded concrete cube model, the proportion of small stone, medium stone, big stone and large stone is $2: 2: 3: 3$. In the mesoscopic numerical model, the average size of $10 \sim 20$ $\mathrm{mm}$ fine aggregate is $15 \mathrm{~mm}$, the average size of $20 \sim 40$ $\mathrm{mm}$ medium aggregate is $30 \mathrm{~mm}$, the average size of $40 \sim$ $80 \mathrm{~mm}$ big aggregate is $60 \mathrm{~mm}$, and the average size 80 $150 \mathrm{~mm}$ large aggregate is $115 \mathrm{~mm}$. Numbers of aggregate are shown in Table 1 .

Table 1. The numbers of spherical aggregates in concrete model.

\begin{tabular}{l|c|c|c|c}
\hline Aggregate size & $10 \sim 20 \mathrm{~mm} /$ fine stone & $20 \sim 40 \mathrm{~mm} /$ medium stone & $40 \sim 80 \mathrm{~mm} /$ big stone & $80 \sim 150 \mathrm{~mm} / \mathrm{large}$ stone \\
\hline Numbers of aggregates & 5503 & 688 & 129 & 18 \\
\hline
\end{tabular}

Table 2. The numbers of polyhedral aggregates in concrete model.

\begin{tabular}{c|c|c}
\hline Aggregate size & $\begin{array}{c}10 \sim 20 \mathrm{~mm} / \\
\text { fine stone }\end{array}$ & $\begin{array}{c}20 \sim 40 \mathrm{~mm} / \\
\text { medium stone }\end{array}$ \\
\hline $\begin{array}{c}\text { Numbers of } \\
\text { aggregates }\end{array}$ & 158 & 20 \\
\hline
\end{tabular}

Figure 4 shows the randomly generated convex polyhedral aggregate model in the cylinder. It can be seen that the random convex polyhedral aggregates are equally filled in the cylindrical specimen, and the aggregate shape is close to the natural shape.

Figure 3. The full-graded spherical aggregate concrete model. 


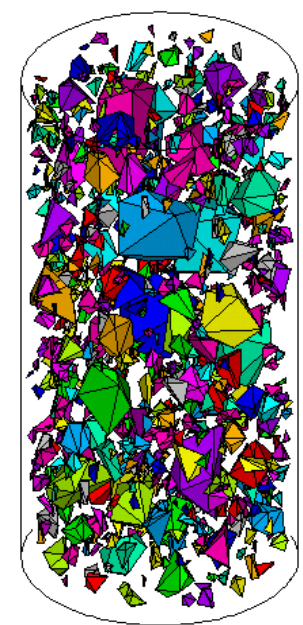

Figure 4. The random convex polyhedral aggregate concrete model.

\section{Conclusions}

This paper studies on three-dimension random aggregate generation of numerical concrete model, the research results are as follows:

(1) The generation of spherical aggregates of concrete mesoscopic model is realized.
(2) Based on the spherical aggregates, the convex polyhedral aggregates of concrete mesoscopic model is generated.

(3) The aggregates are equally distributed in the concrete model and close to natural aggregate shape.

At the same time, the aggregate is simplified as a sphere or a polyhedron, and the concrete model with sphere and convex polyhedral aggregates is obtained. In the future, study on the mesh discretization and the mesoscopic numerical simulation of the concrete model will be carried out.

\section{References}

1. F. H. Wittmann, P. E. Roelfstra and H. Sadouki, Mater. Sci. Eng. 68, 239(1985).

2. Z.Q. Yue, S. Chen and L.G. Tham, Comput. Geotech. 30, 375(2003).

3. H.F. Ma, H.Q. Chen and B.K. Li, J. Hydraul. Eng. 10, 27(2004).

4. G. Cusatis, D. Pelessone and A. Mencarelli, Cement. Concrete. Comp. 33, 881(2011).

5. B. Wang, H. Wang, Z.Q. Zhang and M.J. Zhou, Chin. J. Comput. Mech. 34, 591(2017).

6. W. Qin and C.B. Du, Eng Mech. 29, 186(2012).

7. G. Ma, W. Zhou, X.L. Chang and C.B. Zhou, Chin. J. Geotech. Eng. 33, 746(2011). 